\title{
COMPARISON OF PHYSIOLOGICAL PARAMETERS BETWEEN AMATEUR AND PROFESSIONAL BHARATHANATYAM DANCERS
}

\section{S Saba Ananth ${ }^{1}$}

\section{Abstract}

Physiologists claim to have recognized the therapeutic potential of the pre-eminent classical dance tradition of India known as Bharathanatyam to contribute to the formation of a healthy lifestyle equipped with kinetic, cognitive and spiritual elements of outstanding recreational value. Accordingly, the further a person gets involved in daily practices of Bharathanatyam, the greater it impacts on his or her physiological condition. In this background this study aims at comparing the variations that occur in the physiological parameters of professional and amateur Bharathanatyam dancers respectively, such as resting heart rate (RHR), systolic blood pressure (SBP) and diastolic blood pressure (DBP) and $\mathrm{VO}_{2}$ max. The subjects used for the study were sixty women Bharathanatyam dancers $[(\mathrm{N}=60)$, (age 19-23) (BMI $27 \pm 1.5)$ ] from Jaffna, Sri Lanka. They were classified as professional dancers [(PD) $(n=30$, practicing 90 to $120 \mathrm{~min} /$ day for 5 to 6 day / week over the period of minimum 5 years $)]$ and amateur dancers [(AD) $(n=30$, practicing 30 to $60 \mathrm{~min} / \mathrm{day} / 2$ to 3 day / week over a period of minimum 5 years)]. Taking each of them individually, their resting heart rate (RHR) was measured by palpating the radial artery for one whole minute, their blood pressure (SBP,DBP) was measured, using a standardized sphygmomanometer and stethoscope, and their $\mathrm{VO}_{2}$ max was assessed by means of the Queen's step test. Their resting heart rate and blood pressure were taken during the early morning hours while the collected data were statistically treated by using independent ' $t$ ' tests, with a confidence level of 0.05 fixed to test the significance. The results of the study showed that professional dancers had lesser resting heart rate [(RHR) (mean 68.47), systolic blood pressure (SBP) (mean 113.23), diastolic blood pressure (DBP) (mean 77.17) and higher $\mathrm{VO}_{2} \max$ (mean 46.67)] than amateur dancers [whose RHR (mean 77.93), SBP (mean 117.83), DBP (mean 80.50) and $\mathrm{VO}_{2}$ max (mean 44.33)]. Hence it was concluded that, professional bharathanatyam dance practice positively influence the physiological parameters of the individual in respect of $\mathrm{RHR}, \mathrm{SBP}, \mathrm{DBP}$ and $\mathrm{VO}_{2}$ max than amateur dance practice. In light of the findings, the study attempts to show that a submaximal dancing intensity is adequate for dancers to improve their physiological parameters.

Key words: Bharathanatyam, Blood Pressure, Resting Heart Rate
${ }^{1}$ Sports Science Unit, University of Jaffna, Sri Lanka

saba_ananth@yahoo. com

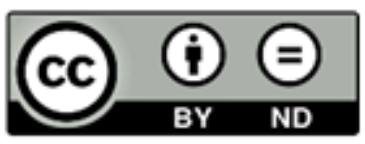

This article is published under the Creative Commons CC-BY-ND License (https://creativecommons.org/licenses/by-nd/4.0/). This license permits use, distribution and reproduction, commercial and non-commercia, provided that the original work is properly cited and is not change anyway. 


\section{INTRODUCTION}

Dance involves the body and the mind both. It is a medium of expression and

communication and a form of nonverbal communication between humans. Gymnastics, figure skating and synchronized swimming are sports that incorporate dance, while martial arts kata are often compared to dances. Motion in ordinarily inanimate objects may also be described as dances, e.g., "the leaves are supposed to dance in the wind". According to (NDTA. 2004), 'Creating dance provides people with the opportunity to make individual responses to their world' of expressive and creative nature. As such, dance is acknowledged as being instrumental in developing young people's creative and artistic, personal and social skills. Moreover, as a physical activity, dance may also impact positively on fitness, health and wellbeing. Much research in sport suggests that physical activity can have a positive effect on physical fitness (Blair SN et.al. 2001, Strong WB.et.al. 2005)

It is discovered that long-term exercise can help to make the muscles expand the lungs stronger and more efficient. This can increase the capacity of the chest to breathe in more air in each breath and increase the number of capillaries around the alveoli, or air sacs, speeding oxygen absorption in the blood. This results in being able to exercise at a higher intensity for a longer period of time.

The pulmonary and circulatory system are responsible for moving blood from the heart to the lungs and back to the heart and getting rid of waste products in the blood while helping to distribute blood rich in oxygen. Exercise can help strengthen and make this system more efficient. Pulmonary functions are generally determined by respiratory muscle strength, compliance of the lung and thoracic cavity, airway resistance and elastic recoil of the lungs (Cotes, 1975).

It is acknowledged that low physical activity and fitness levels are associated with ill health (Blair, 1989). Although there is evidence that exercise can be of benefit to health, it must be regular if these benefits are to be attained/maintained. It is important that regular physical activity is encouraged and that appropriate exercise sessions are promoted. However, the mode of exercise must be acceptable to the target population - that is, the participants must find the activity enjoyable and be able to participate regularly and it may be necessary to show that the exercise prescription is effective in improving fitness levels. The concept of "a little exercise is better than nothing" has been promoted (Mutrie. 1993).

The physiological response to dance is dependent on the intensity, duration and frequency of the exercise as well as the environmental conditions. During dance practice, requirements for oxygen and substrate in skeletal muscle are increased, as are the removal of metabolites and carbon dioxide. Chemical, mechanical and thermal stimuli affect alterations in metabolic, cardiovascular and ventilatory function in order to meet these increased demands.

A good dancer must also possess great coordination, a highly developed kinesthetic 
awareness, control over weight and balance in motion, and endurance is essential to continued existence of prolonged dance performance to develop awareness of space, a strong sense of rhythm, and an appreciation of music. Particularly in theatrical dance, the dancer must be able to project movement clearly and make its expressive qualities intelligible to the audience. Grace, fluidity, and harmony of the body are also frequently desired in the dancer, as is physical beauty.

Bharatanatyam is a very popular dance form in South India, and the oldest of all classical dance forms. The general etymology of Bharathanatyam is BHAva (expression) + RAga (music) + TAla(rhythm) + NATYAM(dance). The variety and style of the dance and musical accompaniment provide to the people tastes in performing them. In India the earliest book discussing dance, the Natya-sastra still survives. This work, which is sacred in Indian culture, codifies dance into a series of rules determining the gestures used to depict different themes and emotions. The Bharathanatyam is a classical dance form based on this treatise. In the modern day scenario, it is performed by both male and female artists. Many learn as a hobby and few make it as a profession. Whether taken as a hobby or a profession it certainly needs a great deal of practice, concentration and dedication.

Despite the fact that the time of motor activity is relatively long in its training sessions (classes), curiously the competitive presentations are performed with intense physical pace and last only three to seven minutes. This usual practice could be considered as a physiological failure, because it probably infringes the specificity of training principle (Flouris et al., 2004).

One single fitness measurement able to predict success in dance will clearly vary depending on numerous variables including age, sex and level of performance. Hence the purpose of the study was to compare physiological parameters between amateur and professional bharathanatyam dancers.

\section{OBJECTIVE OF THE STUDY}

The objective of this study was to compare physiological parameters between amateur and professional bharathanatyam dancers.

\section{Materials and methods}

To achieve the objective of the study sixty $(\mathrm{N}=60)$ women Bharathanatyam dancers were purposively selected from Jaffna, Sri Lanka, and they were classified into Professional Dancers [(PD) $(n=30$, practice bharathanatyam 90 to $120 \mathrm{~min} /$ day for 5 to 6 day / week over the period of minimum 5 years)] and Amateur Dancers[(AD)( $\mathrm{n}=30$, practice bharathanatyam 30 to 60 min / day/ 2 to 3 day / week over the period of minimum 5 years)] and selected as subjects. Their Resting Heart Rate (RHR) was measured using Palpating the radial artery for one full minute for each subject and Blood Pressure [Systolic Blood Pressure (SBP), Diastolic Blood Pressure (DBP)] using Standardized sphygmomanometer and stethoscope and $\mathrm{VO}_{2} \max$ were assessed by Queen's step test. The resting heart rate and blood pressure were taken during early morning hours and the collected data were statistically 
treated by using independent ' $t$ ' test. The level of confidence adjusted to 0.05 in order to test the significance of the approach.

\section{RESULTS}

Table-1: Comparison of Rhr, Sbp, Dbp Between Professional and Amateur Female Bharathanatyam Dancers.

\begin{tabular}{cccccc}
\hline Variable & Group & Mean & SD & SE & t-value \\
\hline $\begin{array}{c}\text { RHR } \\
\text { (b/min) }\end{array}$ & PD & 68.47 & 3.81 & 0.70 & \\
SBP & AD & 77.93 & 8.32 & 1.52 & $5.67^{*}$ \\
$(\mathrm{mmHg})$ & PD & 113.23 & 5.56 & 1.01 & \\
& AD & 117.83 & 5.03 & 0.92 & $3.36^{*}$ \\
DBP & PD & 77.17 & 5.20 & 0.95 & \\
$(\mathrm{mmHg})$ & AD & 80.50 & 2.73 & 0.50 & $3.12^{*}$ \\
& PD & 46.67 & 2.57 & 0.47 & \\
$\mathrm{VO}_{2} \mathrm{max}$ & & & & & \\
& AD & 44.33 & 2.98 & 0.54 & \\
\hline
\end{tabular}

*Significant at .05 level of confidence with $\mathrm{df}(1,58)$ is 2.00

The results of the study shows that Professional Dancers had less Resting Heart Rate (RHR) (mean 68.47), Systolic Blood Pressure (SBP) (mean 113.23), Diastolic Blood Pressure (DBP) (mean 77.17) and higher $\mathrm{VO}_{2} \max$ (mean 46.67) than Amateur Dancers whose physiological parameters are often calculated as RHR (mean 77.93), SBP (mean 117.83), DBP (mean 80.50) and $\mathrm{VO}_{2}$ max (mean 44.33). Hence it was concluded that, Professional
Bharathanatyam Dancers have Lower RHR, SBP, and DBP than Amateur Dancers.

\section{DISCUSSION}

Physical inactivity and low cardiorespiratory fitness are recognized as important causes of morbidity and mortality (Twisk, et-al. 1998). It is generally accepted that people with higher levels of physical activity tend to have higher levels of fitness and that physical activity can improve 
cardio-respiratory fitness (Burchfiel, et-al. 1997).

Dance is a predominantly intermittent type of exercise. In choreographed dance, training is very demanding and energetic physical movement. In the present study, it was found that all the physiological parameters of the professional bharathanatyam dancers were significantly better than amateur dancers.

Dance is an art form that generally refers to movement of the body, usually rhythmic and to music, used as a form of expression, social interaction or presented in a spiritual or performance setting. The Dance movements may be without significance in both classical dance and folk dance. Bharathanatyam is a salient feature of the aesthetic, artistic and creative form of dance. It is sacred in Indian culture, codified by a series of gestural rules to depict different themes and emotions.

It may indicate that the associated dance training outcomes could be affected by variations in duration, intensity and frequency they undergo. Regular dance training is essential for maintain and developing the dancer's technique and coordination. The energy demands that arise during the training sessions stand in rather sharp contrast with those that prevail during stage performances. The result shows that the professional dancers have better RHR and BP, compared with amateur dancers.

The literature indicates that changes in cardiorespiratory endurance, $\mathrm{VO}_{2} \max$ are directly related to the subject's initial fitness level and the frequency, intensity and duration of the training programme. Some aerobic type activities have a close association with $\mathrm{VO}_{2}$ max (Hemple and wells, 1985). It has been shown that arm work performed above the head produces a higher $\mathrm{VO}_{2}$ max than the work performed bellow head level, due to an increased sympathetic tone (Parker et-al 1989). According to Hamilton et.al (1989), aerobic dance and circuit training can be intense enough to promote aerobic capacity. In another study, improvement in cardiovascular fitness is related to the mode, frequency, duration, intensity, and rate of progression of exercise (Kirkendall \& Calabrese, 1983). The data suggest that dance is an activity for promoting fitness and will improve aerobic and physical working capacity. In the present investigation, it was discovered the Professional Dancing group has lower resting heart rate (RHR) and blood pressure (BP) than the Amateur Dancing group. It revealed that professional dance groups were able to have more powerful and more effective body functions than amateur dance groups.

\section{CONCLUSION}

From the results it was clear that, Professional Dancers were better than Amateur Dancers on Resting Heart Rate, Blood Pressure and $\mathrm{VO}_{2}$ max. Hence it was concluded that professional dance (PD) practice may have positive influence on health status in respect to cardiac function as well as blood pressure in women dancers. In light of this kind of study, attention is required to increase the understanding of the artistic and athletic needs of the dancers in different genres, with a focus on their requirement to cope physiologically theater demands of extreme nature for rigorous body movements. 


\section{REFERENCES}

Blair S.N, Cheng Y, and Holder, S. (2001). Is physical activity or physical fitness more important in defining health related benefits? Medicine and Science in Sport and Exercise.pp.379398.

Blair S.N, Kohl H.W. and Paffenbarger R.S., et al. (1989). Physical fitness and all-cause mortality. A prospective study of healthy men and women. JAMA; 262:2395-401.

Burchfiel C.M., Enright P.L. and Sharp D.S.. (1997). Factors Associated with Variations in Pulmonary Functions among Elderly Japanese-American Men. Chest; 112:87-97.

Cotes J.E. (1975). Lung function assessment and application in Medicine. 3rd ed. Oxford: Blackwell Scientific publications.

Flouris A.D., Koutedakis Y., Nevill A., Metsios G.S., Tsiotra G. and Parasiris Y. (2004). Enhancing specificity in proxy-design for the assessment of bioenergetics. J. Sci. Med. Sport, 7: 197-204.

Hamilton, S. J., Patterson, P., \& Sucec, A. (1989). Physiological benefits of continuous versus interval circuit aerobic dance exercise. Unpublished manuscript, San Diego State University, Physical Education, SanDiego. 19(5).36 -45.
Hempel, L.S., \& Wells, C.L. (1985). Cardiorespiratory cost of the Nautilus Express Circuit. The Physician and Sports medicine. (4), 82-97.

Kirkendall D.T. \& Calabrese L.H. (1983). 'Physiological aspects of dance'". Clin sports med 2 (3): 525-37.

Mutrie N, Blamey A, Davison R, et al. (1993). Class based and homebased activities for older people. Research digest no 30, research report no 32. Edinburgh: Scottish Sports Council.

National Dance Teachers Association (NDTA). Maximising Opportunity, Policy Paper.

Parker, S.B., Hurley, B.F., Ranlon, D.P., \& Vaccaro, P. (1989). Failure of target heart rate to accurately monitor intensity during aerobic dance. Medicine and Science in sports and Exercise, 21:230-234.

Strong W.B., Malina R.M., Blimke C.J.R., Daniels S.R., et. al. (2005). Evidence based physical activity for school-age youth. Journal of Pediatrics.146: 732737.

Twisk J.W., Staal B.J., Brinkman M.N. (1998). Tracking of Lung Function Parameters and the Longitudinal Relationship with Lifestyle. European Respiratory Journal.; 12:627-634. 